\title{
Correlation of Clinicopathological Features and IL6 Expression in Tumor Budding of Colon Adenocarcinoma
}

\section{Koichi Sato}

Shinshu Ueda Medical center

Takeshi Uehara ( $\nabla$ tuehara@shinshu-u.ac.jp)

Shinshu University School of Medicine https://orcid.org/0000-0002-7694-9015

\section{Mai Iwaya}

Shinshu Daigaku

\section{Tomoyuki Nakajima}

Shinshu Daigaku

\section{Yosuke Tobe}

Shinshu Daigaku

\section{Yusuke Miyagawa}

Shinshu Daigaku

\section{Hiroyoshi Ota}

Shinshu Daigaku

\section{Research article}

Keywords: interleukin-6; tumor budding; RNA in situ hybridization; colon adenocarcinoma

Posted Date: May 26th, 2020

DOI: https://doi.org/10.21203/rs.3.rs-26146/v1

License: (9) This work is licensed under a Creative Commons Attribution 4.0 International License. Read Full License 


\section{Abstract}

Background: Interleukin-6 (IL6) is one of the main cytokines produced by cancer-associated fibroblasts (CAFs). IL6 is linked with cancer progression and poor prognosis by activating cancer cells and modifying the cancer microenvironment. However, little is known about the expression of IL6 in tumor budding (TB) and its association with TB in colon adenocarcinoma (CA).

Methods: The clinicopathological and prognostic significance of IL 6 in TB was examined using a tissue microarray consisting of 36 patient samples of TB in CA. IL 6 mRNA was detected by RNAscope kit. Patients were stratified into negative and positive $I L 6$ expression groups.

Results: IL6 expression was overwhelmingly observed in CAFs but was negligible in cancer cells. In the IL6-positive group in CAFs, TB grade was higher than in the IL6-negative group ( $P=0.0161)$. There was a significant difference in overall survival (OS) between CA cases in the IL6-positive group and the IL 6 negative group (log rank test, $P=0.0367$ ). Cox proportional hazard regression model revealed that the IL6negative group (OR $=0.25 ; 95 \% \mathrm{Cl}: 0.05-0.96 ; P=0.0440)$ had better OS for $\mathrm{CA}$ than the IL 6 -positive group.

Conclusions: TB may be affected by IL 6 expression, and IL 6 expression in CAFs at TB may make $I L 6$ an important prognostic marker.

\section{Background}

Colorectal cancer (CRC) has increasing morbidity and mortality worldwide and is a global health problem [1]. Despite the high prevalence of colorectal cancer, the pathological mechanisms remain largely unknown [2]. However, many prognostic factors for colorectal cancer have been studied. In particular, the tumor budding (TB) region is a unique site and is known to be deeply involved in metastasis and invasion [3]. It has been demonstrated that TB is involved in EMT, which is known to be affected by the surrounding microenvironment of cancer $[4,5]$. Cancer-associated fibroblasts (CAFs) have an important role in the cancer microenvironment, and IL6 produced by CAFs is involved in various processes [6]. We focused on the microenvironment in TB. IL6 is an important cytokine but has not been studied in TB. We investigated the clinicopathological characteristics of IL 6 expression using RNAscope, a recently developed ISH technique with high sensitivity.

\section{Methods}

\section{Patients and materials}

This study was conducted in accordance with the Declaration of Helsinki and was approved by the ethics committee of Shinshu University School of Medicine (approval no. 4088). Among 115 colon adenocarcinoma (CA) cases surgically resected at Shinshu University Hospital, Matsumoto, Japan between 2010 and 2012, stage I-III cases with TB were selected. Clinicopathological data were obtained from medical records. Materials used for evaluation were archived formalin-fixed paraffin-embedded 
tissues. According to the report of Lugli et al., TB was classified into Bd1 ( $0-4$ buds), Bd2 (5-9 buds), and $B d 3$ ( $\geq 10$ buds) [3]. Furthermore, $B d 1$ and $B d 2$ were defined as low-grade TB and $B d 3$ was defined as high-grade TB. In the budding area, the score of inflammatory cell infiltration (tumor-infiltrating lymphocytes, TILs) was measured. According to the report of Ropponen et al., the TIL scores were: none, 0 ; mild, 1; moderate, 2; and marked, 3[7]. TIL scores were classified into low-grade scores 0 and 1 and high-grade scores 2 and 3.

\section{TMA construction}

A tissue microarray (TMA) was prepared from paraffin blocks containing sufficient tumor. The TMA was $3 \mathrm{~mm}$ in diameter and contained a fully analyzable TB region. The TB region was defined as an area with a single cell or a detached group of tumor cells consisting of five cells or fewer, and was selected based on the morphology of the hematoxylin and eosin (H\&E)-stained slide [8]. The generation of the TMA was in accordance with our previous report [9].

\section{IL6 RNA in situ hybridization}

IL6 mRNA was detected using an RNAscope kit (Advanced Cell Diagnostics, Hayward, CA, USA), as previously described [9]. Intracellular brown dots indicated positive staining. IL 6 expression was measured according to a 5-grade scoring system recommended by the manufacturer's protocol. The 5grade scoring system was determined under a 20x objective lens as follows: no staining, $0 ; 1-3$ dots/cell, 1+; 4-10 dots/cell, 2+; 10-15 dots/cell, 3+; and >15 dots/cell, defined as 4+. IL 6 mRNA expression was defined as negative expression in grade $0,1+$, and $2+$, and positive expression in grade $3+$ and $4+$.

\section{Statistical analysis}

Pearson's chi-squared test, log-rank test, and Cox proportional hazard regression analysis were analyzed by JMP Statistics software version 13 (JMP, Tokyo, Japan). A P-value less than 0.05 was considered significant.

\section{Results}

\section{IL6 expression in cancer stroma}


In the TB region, IL6-expressing cells were mainly identified in cancer stroma. These IL6-expressing cells were spindle-shaped and were considered as CAFs (Fig. 1A, 1C). In four cases, IL 6 expression could not be detected in the cancer stroma. Thirteen cases could be recognized as the IL 6 -high expression group. There was no tendency in the distribution of expressing cells in the stroma. However, there was almost no IL6 expression in the cancer cells in the TB region. Cancer cells throughout the TMA core also had little $I L 6$ expression. Thirty cases were completely negative for $I L 6$ expression in cancer cells. IL 6 expression in the cancer cells was faint and had no characteristic distribution. No cases could be recognized as IL6positive.

\section{Association between IL6 expression and clinicopathological characteristics}

As presented in Table 1, the clinicopathological characteristics of patients with CA are described in Table 1. In the IL6-positive group, TB grade was higher than in the IL6-negative group $(P=0.0161)$. There was no significant difference between the IL 6 -positive group and the IL 6 -negative group in terms of age, sex, vascular invasion, histological grade, TILs, or TNM stage. 
Table 1

IL6 expression and clinicopathological characteristics in CA.

\begin{tabular}{|c|c|c|c|c|}
\hline & & IL6 expression & & \\
\hline Factors & $\mathrm{n}$ & Positive $(n=13)$ & Negative $(n=23)$ & $P$-value \\
\hline Age & & & & 0.9231 \\
\hline$>70$ years & 17 & 6 & 11 & \\
\hline$\leq 70$ years & 19 & 7 & 12 & \\
\hline Sex & & & & 0.8767 \\
\hline Male & 16 & 6 & 10 & \\
\hline Female & 20 & 7 & 13 & \\
\hline TILs & & & & 0.9685 \\
\hline High & 22 & 8 & 14 & \\
\hline Low & 14 & 5 & 9 & \\
\hline Histological grade & & & & 0.587 \\
\hline High & 20 & 8 & 12 & \\
\hline Low & 16 & 5 & 11 & \\
\hline Vascular invasion & & & & 0.7286 \\
\hline High & 18 & 7 & 11 & \\
\hline Low & 18 & 6 & 12 & \\
\hline Tumor budding grade & & & & $0.0161^{*}$ \\
\hline High & 3 & 3 & 0 & \\
\hline Low & 33 & 10 & 23 & \\
\hline TNM stage & & & & 0.587 \\
\hline$|-| \mid$ & 20 & 8 & 12 & \\
\hline III & 16 & 5 & 11 & \\
\hline
\end{tabular}

\section{IL6negativity predicts better prognosis of CA}


To clarify the impact of IL 6 expression, Kaplan-Meier analysis with log-rank test was used to evaluate the association between IL6 expression and OS in CA (Fig. 2). The IL6-negative group (median OS, 1980 (range, 1771-2531) days) had significantly better OS than the IL6-positive group (median OS, 1556 (range; 1212-2377.5) days) (log-rank test, $P=0.0367$ ).

A Cox proportional hazard regression model revealed the relationship between clinicopathological factors and OS (Table 2). These results revealed that the IL 6-negative group $(\mathrm{OR}=0.25 ; 95 \% \mathrm{Cl}: 0.05-0.96 ; P=$ 0.0440 ) had better OS for CA than the IL6-positive group.

Table 2

Univariate analyses for prognostic factors of CA.

\begin{tabular}{|lll|}
\hline Factors & Univariate analysis & \\
\hline & OR $(95 \% \mathrm{Cl})$ & $P$-value \\
\hline Age: $>70$ years vs $\leq 70$ years & $2.82(0.74-13.38)$ & 0.1291 \\
\hline Sex: male vs female & $3.36(0.88-15.95)$ & 0.0753 \\
\hline Histological grade: low vs high & $0.33(0.05-1.37)$ & 0.135 \\
\hline TILs: low vs high & $3.53(0.93-16.77)$ & 0.0638 \\
\hline Vascular invasion: absent vs present & $0.822(0.20-3.11)$ & 0.7701 \\
\hline Tumor budding grade: low vs high & $0.79(0.14-14.58)$ & 0.8253 \\
\hline TNM stage: I-II vs III & $1.01(0.27-4.08)$ & 0.9883 \\
\hline IL6 expression: negative vs positive & $0.25(0.05-0.96)$ & $0.044 *$ \\
\hline Asterisk $\left(^{*}\right)$ indicates a significant difference between groups $(P<005)$. \\
\hline
\end{tabular}

\section{Discussion}

In the present study, we demonstrated that IL6 expression in TB had significant effects on OS. Recently, it has been shown that CAFs, which account for the majority of the tumor stroma, have an important role in producing factors involved in invasion and metastasis. In CRC, CAFs are known to be involved in prognostic factors such as invasion and metastasis [10] [11], and there are some reports of IL6 expression in CAFs [12] [13]. Hugo et al. reported that cancer cells cause an inflammatory response in fibroblasts and promote IL 6 expression [14]. In our study, no association was found between IL 6 and inflammation expressed as TILs, possibly because of the method of evaluation and the number of cases. However, there are no reports of IL 6 expression in CAFs in CRC. Nonetheless, there are reports of IL 6 expression from CAFs in several other carcinomas [15] [16]. Qiao et al. reported that IL6 expression from CAFs is associated with poor prognosis in esophageal squamous cell carcinoma [16]. This is the first report on IL6 expression from CAFs in the TB region, and indicates that IL6 expression is a poor prognostic factor. 
TB grade was previously reported to be associated with prognosis [17]. In our study, TB grade was not related to prognosis, possibly because of the small number of samples. The TB region strongly affects metastasis and invasion. Although the mechanism of TB involvement in prognosis is unclear, the involvement of EMT has been reported in recent years [5]. TB in CRC has been shown to upregulate mesenchymal markers and known inducers of EMT, such as the transcription factors ZEB1 and ZEB2 [18]. However, another report revealed that TB shows downregulation of E-cadherin but does not share other regulatory changes common to EMT, suggesting that TB formation may occur by other mechanisms [19] [20]. Yamada et al. reported that ZEB1, an EMT protein, is highly expressed in stroma near TB [20]. Our study demonstrates that IL6 expression is correlated with TB grade. As mentioned above, its involvement of TB and EMT is speculated [20]. EMT and IL6 expression in the cancer stroma are known to be involved in miR-34A suppression [21]. This fact proves an indirect link between TB and IL6. However, IL6-affected TB may be directly involved in EMT IL6-affected TB may be directly involved in EMT.

There are several studies of IL 6 in CRC, but these mostly focused on IL6 expression in cancer cells [22] [23]. Although many reports indicate that IL6 expression in cancer cells is associated with poor prognosis [24] [25], one report demonstrated that IL6 expression at other sites confers a favorable prognosis [26]. Meanwhile, Nagasaki et al. reported that IL6 expression is higher in CAFs than in cancer cells when comparing cancer cells and CAFs isolated from human CRC [12]. In our study, IL6 expression has been largely identified in the stroma corresponding to CAFs, and IL6 expression in cancer cells is negligible. Therefore, although IL 6 produced by CAFs seems to have a strong effect on prognosis, further investigation is necessary. Many reports have examined IL6 expression by immunostaining [24] [25] [26], but there may be many nonspecific reactions. Thus, RNA in situ measurement may provide more accurate information.

There are several limitations of our study. An increased number of cases would enable more accurate information to be obtained. In addition, expression analysis of $I L 6$ receptor in cancer cells in the TB area should be performed.

Taken together, inhibition of IL 6 expression may be a potential therapeutic strategy for the treatment of cancers in which IL 6 from CAFs may have important effects.

\section{Conclusions}

Our results reveal the relationship between IL 6 expression of CAFs and TB in CA. A further study is warranted to confirm these findings.

\section{Abbreviations}

IL6, interleukin-6; CAFs, cancer-associated fibroblasts; TB, tumor budding; CA, colon adenocarcinoma 


\section{Declarations}

\section{Ethics approval and consent to participate}

This study was approved by the ethics committee of Shinshu University School of Medicine (Approval Code: 4088). The requirement of informed consent was waived, and an opt-out method was used due to the retrospective design of the study. The investigation was conducted in compliance with the Helsinki Declaration.

\section{Consent for publication}

Not applicable.

\section{Availability of data and materials}

All data generated and analyzed during the current study are available from the corresponding author on reasonable request.

\section{Competing interests}

The authors declare that they have no competing interests.

\section{Funding}

This study was partially supported by the Hokuto Foundation for Bioscience (grant awarded to T.U.). The funding body had no role in the study design, collection, analysis, or interpretation of data, or manuscript writing. The authors declare no conflicts of interest.

\section{Authors' contributions}

KS participated in the design of the study, performed the pathological analysis, and drafted the manuscript. TU and MI helped with the pathological analysis. TU performed statistical analysis. TN and YT conducted immunohistochemistry. KS and YM examined the clinical data of cases. HO and TU critically revised the draft for important intellectual content. All authors have read and approved the manuscript.

\section{Acknowledgements}

We are grateful to Yukihoro Kobayashi, Masanobu Momose, Yasuyo Shimojo, Naoko Ogiwara, Akiko Inamura, Chitoshi Arai, Yasuhiro Kinugawa, Marina Nuno, Kanade Wakabayashi, Naoko Yamaoka, and Tomoya Hachisu at Shinshu University Hospital for their excellent technical assistance. We thank H. Nikki March, PhD, from Edanz Group (www.edanzediting.com/ac) for editing a draft of this manuscript.

\section{References}


1. Bray F, Ferlay J, Soerjomataram I, Siegel RL, Torre LA, Jemal A Global cancer statistics 2018: GLOBOCAN estimates of incidence and mortality worldwide for 36 cancers in 185 countries. CA Cancer J Clin. 2018.

2. Tariq K, Ghias K Colorectal cancer carcinogenesis: a review of mechanisms. Cancer Biol Med. 2016;13:120-135.

3. Lugli A, Kirsch R, Ajioka Y, Bosman F, Cathomas G, Dawson Het al. Recommendations for reporting tumor budding in colorectal cancer based on the International Tumor Budding Consensus Conference (ITBCC) 2016. Mod Pathol. 2017;30:1299-1311.

4. Ueno H, Shinto E, Shimazaki H, Kajiwara Y, Sueyama T, Yamamoto Jet al. Histologic categorization of desmoplastic reaction: its relevance to the colorectal cancer microenvironment and prognosis. Annals of surgical oncology. 2015;22:1504-1512.

5. Zlobec I, Lugli A Epithelial mesenchymal transition and tumor budding in aggressive colorectal cancer: tumor budding as oncotarget. Oncotarget. 2010;1:651-661.

6. Sanchez-Lopez E, Flashner-Abramson E, Shalapour S, Zhong Z, Taniguchi K, Levitzki Aet al. Targeting colorectal cancer via its microenvironment by inhibiting IGF-1 receptor-insulin receptor substrate and STAT3 signaling. Oncogene. 2016;35:2634-2644.

7. Ropponen KM, Eskelinen MJ, Lipponen PK, Alhava E, Kosma VM Prognostic value of tumourinfiltrating lymphocytes (TILs) in colorectal cancer. J Pathol. 1997;182:318-324.

8. Lugli A, Karamitopoulou E, Zlobec I Tumour budding: a promising parameter in colorectal cancer. $\mathrm{Br}$ J Cancer. 2012;106:1713-1717.

9. Nakajima T, Uehara T, Maruyama Y, Iwaya M, Kobayashi Y, Ota H Distribution of Lgr5-positive cancer cells in intramucosal gastric signet-ring cell carcinoma. Pathol Int. 2016;66:518-523.

10. Tommelein J, Verset L, Boterberg T, Demetter P, Bracke M, De Wever O Cancer-associated fibroblasts connect metastasis-promoting communication in colorectal cancer. Front Oncol. 2015;5:63.

11. Son GM, Kwon MS, Shin DH, Shin N, Ryu D, Kang CD Comparisons of cancer-associated fibroblasts in the intratumoral stroma and invasive front in colorectal cancer. Medicine (Baltimore). 2019;98:e15164.

12. Nagasaki T, Hara M, Nakanishi $H$, Takahashi $H$, Sato $M$, Takeyama $H$ Interleukin- 6 released by colon cancer-associated fibroblasts is critical for tumour angiogenesis: anti-interleukin-6 receptor antibody suppressed angiogenesis and inhibited tumour-stroma interaction. Br J Cancer. 2014;110:469-478.

13. Tsai KS, Yang SH, Lei YP, Tsai CC, Chen HW, Hsu CYet al. Mesenchymal stem cells promote formation of colorectal tumors in mice. Gastroenterology. 2011;141:1046-1056.

14. Hugo HJ, Lebret S, Tomaskovic-Crook E, Ahmed N, Blick T, Newgreen DFet al. Contribution of Fibroblast and Mast Cell (Afferent) and Tumor (Efferent) IL-6 Effects within the Tumor Microenvironment. Cancer Microenviron. 2012;5:83-93.

15. Higashino N, Koma YI, Hosono M, Takase N, Okamoto M, Kodaira Het al. Fibroblast activation protein-positive fibroblasts promote tumor progression through secretion of CCL2 and interleukin- 6 in esophageal squamous cell carcinoma. Lab Invest. 2019;99:777-792. 
16. Qiao Y, Zhang C, Li A, Wang D, Luo Z, Ping Yet al. IL6 derived from cancer-associated fibroblasts promotes chemoresistance via CXCR7 in esophageal squamous cell carcinoma. Oncogene. 2018;37:873-883.

17. Maffeis V, Nicole L, Cappellesso R RAS, Cellular Plasticity, and Tumor Budding in Colorectal Cancer. Front Oncol. 2019;9:1255.

18. De Smedt L, Palmans S, Andel D, Govaere O, Boeckx B, Smeets Det al. Expression profiling of budding cells in colorectal cancer reveals an EMT-like phenotype and molecular subtype switching. Br J Cancer. 2017; 116:58-65.

19. De Craene B, Berx G Regulatory networks defining EMT during cancer initiation and progression. Nat Rev Cancer. 2013;13:97-110.

20. Yamada N, Sugai T, Eizuka M, Tsuchida K, Sugimoto R, Mue Yet al. Tumor budding at the invasive front of colorectal cancer may not be associated with the epithelial-mesenchymal transition. Hum Pathol. 2017;60:151-159.

21. Rokavec M, Oner MG, Li H, Jackstadt R, Jiang L, Lodygin Det al. IL-6R/STAT3/miR-34a feedback loop promotes EMT-mediated colorectal cancer invasion and metastasis. J Clin Invest. 2014;124:18531867.

22. Zeng J, Tang ZH, Liu S, Guo SS Clinicopathological significance of overexpression of interleukin-6 in colorectal cancer. World J Gastroenterol. 2017;23:1780-1786.

23. Cui G, Yuan A, Sun Z, Zheng W, Pang Z IL-1beta/IL-6 network in the tumor microenvironment of human colorectal cancer. Pathol Res Pract. 2018;214:986-992.

24. Liang B, Li L, Miao R, Wang J, Chen Y, Li Zet al. Expression of Interleukin- 6 and Integrin alphanubeta6 in Colon Cancer: Association with Clinical Outcomes and Prognostic Implications. Cancer Invest. 2019;37:174-184.

25. Chung YC, Chaen YL, Hsu CP Clinical significance of tissue expression of interleukin-6 in colorectal carcinoma. Anticancer Res. 2006;26:3905-3911.

26. Ahmad N, Ammar A, Storr SJ, Green AR, Rakha E, Ellis IOet al. IL-6 and IL-10 are associated with good prognosis in early stage invasive breast cancer patients. Cancer Immunol Immunother. 2018;67:537549 .

\section{Figures}



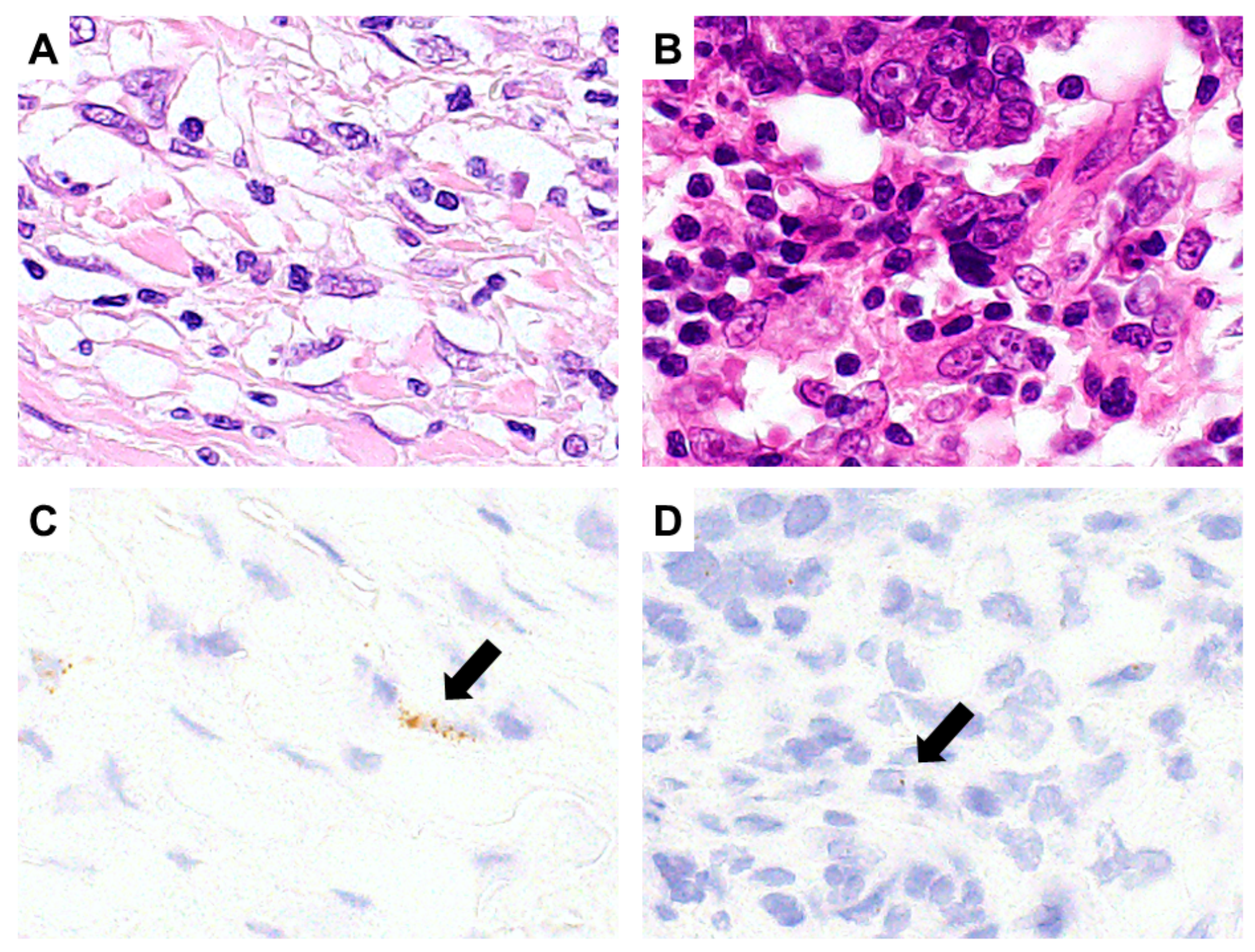

\section{Figure 1}

Representative features of IL6 expression. Representative features of IL6 expression in CAFs (A and C). High levels of IL6 expression (arrow) were determined as IL6-positive. Representative features of IL6 expression in cancer cells at TB (B and D). Faint IL6 expression in cancer cells (arrow) was determined as IL6-negative. (A and B: HE; C and D: IL6 RNAscope) 

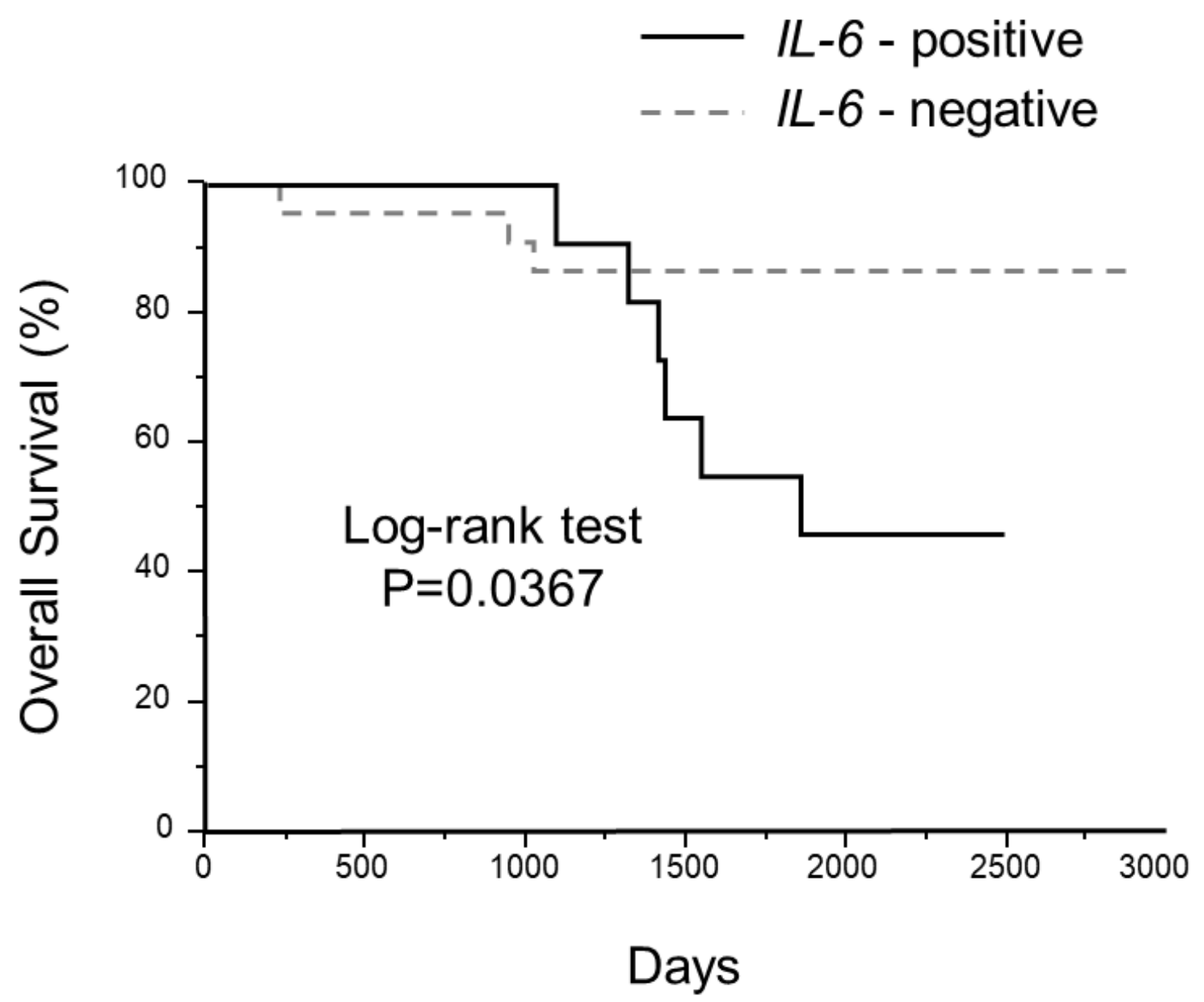

Figure 2

Prognostic value of IL6 in CA by Kaplan-Meier analysis. There was a significant difference in OS between CA cases in the IL6-positive group (median OS, 1556 (range, 1212-2377.5) days) and the IL6-negative group (median OS, 1980 (range, 1771-2531) days) (log rank test, $\mathrm{P}=0.0367$ ). 